\title{
A FULL EXPLANATION OF BEHAVIOR REQUIRES ATTENTION TO TINBERGEN'S FOUR LEVELS OF ANALYSIS
}

\author{
Stefan M. M. Goetz \\ Department of Psychology, Wayne State University, MI, USA \\ stefan.goetz@wayne.edu
}

A Review of the Book

Exploring Animal Behavior: Readings from American Scientist, $6^{\text {th }}$ Edition

Edited by Paul W. Sherman and John Alcock. 2013.

Sinauer Associates, Inc., Massachusetts, 374 pages.

ISBN 978-1-60535-195-7 (Paperback, \$34.95)

Like the five previous editions of Exploring Animal Behavior, this new edition of the anthology is comprised of articles selected from the magazine, American Scientist published by Sigma Xi. The collection contains 37 articles organized into six sections each prefaced with an introduction by the editors Paul Sherman and John Alcock; these section introductions serve the useful purpose of orienting the reader to the overarching themes, and highlight controversies and unanswered questions. Additionally, they provide a brief summary of each article.

The sections serve to illustrate how scientific research on animal behavior is benefitted by and can be pursued both at the proximate (how) level of analysis and at the ultimate (why) level of analysis using Tinbergen's four levels of explanation: mechanism, ontogeny, adaptive value, and phylogeny. In this respect, Sherman and Alcock have excelled at selecting and organizing the articles into sections which demonstrate each type of analysis. Part one, Doing Science and Study Behavior begins 
with three papers which paint a picture of the cultural practices and motives of a behavioral scientist while the last three papers serve as an introduction to, and example of Tinbergen's four levels of analysis; in particular, the paper by Holekamp and Sherman on ground squirrel dispersal behavior most clearly lays out how scientific inquiry can be pursued at each level of analysis.

Both parts two, The Adaptive Value of Social Behavior, and three, The Adaptive Value of Reproductive Behavior, contain papers illustrating the type of questions that are pursued at the functional level of analysis. Part two, introduces readers the "Darwinian puzzle" of providing a functional explanation for altruistic behavior and introduces readers to the controversial theory of multilevel selection theory. In the section introduction, Sherman and Alcock invite readers to try and reinterpret the kin selection and reciprocal altruism explanations of altruistic behavior given in several of the other articles in terms of multilevel selection theory. Part three switches the focus from altruism to reproductive behavior and the role that sexual selection plays in producing adaptions. The section contains an eclectic mix of papers, ranging from sex differences in insects to human mating strategies.

Part four, The Evolutionary History of Behavior, contains five papers that demonstrate the phylogenetic approach to studying behavior. Behaviors of course do not fossilize, nevertheless, the papers included in this section aptly demonstrate how a phylogenetic approach can be conducted with respect to behavior by comparing behaviors in extant species. For example, the paper by Gerald Borgia on bower building behaviors in bowerbirds, constructs (though not explicitly) a phylogenetic tree based on the types and complexity of bowers built by extant bowerbird species.

Part five, The Mechanisms of Behavior, contains five papers which serve to illustrate how adaptations are instantiated within an organism. These include developmental causes as well as internal mechanisms such as genes, neuronal systems, and hormones. Indeed, a common approach to studying mechanisms of behavior taken by behavioral biologists is to explore the role of hormones in regulating behavior. This was well illustrated in the paper by John Wingfield on his now famous Challenge Hypothesis which proposes that testosterone elevations observed in male birds during the breeding season functions to facilitate aggressive behavior.

The final section, Communication Behavior at Four Levels of Analysis, includes six articles which serve to reinforce the message of the anthology, mainly, that the study of behavior should be pursued at all four levels of analysis in order to reach a full understanding.

The papers and section prefaces are written in such a way that they are accessible to scientifically literate readers. Furthermore, the aesthetics of the anthology are excellent; most of the papers include colorful artwork and informative figures that aid greatly in grasping the key empirical findings. Regarding the content, one thing that was a bit disappointing about the anthology was that it did not contain a separate section on the ontology of adaptive traits; nevertheless, the ontological level of analysis was present in many of the papers.

The anthology provides an excellent introduction to the field of behavioral biology and should be of interest to anyone interested in gaining an evolutionary understanding 
of animal behavior and the types of questions and methods used in the field. Moreover, psychologists and graduate students interested in taking a comparative and or an ethological approach will find this anthology a useful starting point for identifying the key figures in the field, modern controversies (e.g., Wilson and Wilson's multilevel selection theory), and areas of study that are ripe for further research. The anthology could also prove useful for academics interested in designing a course that integrates an evolutionary perspective.

\section{ABOUT THE AUTHOR}

Stefan Goetz is a graduate student in the Psychology Department at Wayne State University under the advisement of Dr. Glenn Weisfeld. He received his M.A. degree in Psychology from Wayne State University in 2015 and has a B.A. in German and a B.S. in Psychology from Grand Valley State University. Stefan is interested in honest signaling mechanisms and their hormonal underpinnings used in the context of competitive interactions in both men and women. 